\title{
The intraocular CD4/CD8 ratio in a black South African patient with Behçet's disease uveitis
}

\author{
A Makgotloe, MB BCh, FC Ophth, MMed \\ Division of Ophthalmology, Faculty of Health Sciences, University of the Witwatersrand, Johannesburg, South Africa
}

Corresponding author: A Makgotloe (makgotloe@gmail.com)

\begin{abstract}
A 33-year-old black South African male patient was referred to our uveitis service with a 5-year history of panuveitis refractory to treatment. He had an associated history of recurrent mouth and genital ulcers, predating the uveitis history by about 2 years. The measurement of his intraocular CD4/CD8 ratio helped make a diagnosis of Behçet's disease uveitis.
\end{abstract}

S Afr J Med 2017;107(1):41-42. DOI:10.7196/SAMJ.2017.v107i1.12039

Behçet's disease (BD) is a systemic inflammatory disease of unknown origin. ${ }^{[1]}$ Its diagnosis is based on the recognition of its clinical features as defined by the international criteria for Behçet's disease. ${ }^{[2]}$ These include recurrent oral ulcers with one of the following: penile ulcers, uveitis, skin lesions or positive pathergy test. BD is uncommon in sub-Saharan Africa (SSA), making its diagnosis delayed in most cases ${ }^{[3]}$ I present a case of bilateral panuveitis caused by BD with a delayed diagnosis of about 5 years. Intraocular CD4/CD8 ratio was used to help make the diagnosis of Behçet's uveitis. Measurement of the intraocular $\mathrm{CD} 4 / \mathrm{CD} 8$ ratio could aid in the diagnosis of $\mathrm{BD}$ in countries where BD is less endemic, such as SSA countries.

\section{Case report}

A 33-year-old black South African (SA) male patient was referred to our uveitis service with a 5 -year history of panuveitis refractory to corticosteroids (oral/regional) and methotrexate $20 \mathrm{mg} / \mathrm{week}$. He had an associated history of recurrent mouth and genital ulcers, predating the uveitis history by about 2 years. His visual acuity was light perception without projection on the right eye and no light perception (NLP) on the left eye. He had pigmented keratic precipitates and hypopyon in both eyes. He had bilateral posterior synechiae and no associated iris atrophy. He was bilaterally pseudophakic and intraocular pressure was $10 \mathrm{mmHg}$ and $8 \mathrm{mmHg}$ on the right and left eye, respectively. He had severe vitritis and extensive retinal vasculitis bilaterally, with associated chronic retinal detachment on the left side.

Systemic examination revealed oral and penile ulcers. He had multiple papulomacular skin lesions over his entire body.

The white cell count (WCC) was normal and erythrocyte sedimentation rate (ESR) elevated at $60 \mathrm{~mm} /$ hour. Serum Treponema pallidum particle agglutination assay (TPHA) and rapid plasma reagin (RPR) were non-reactive and toxoplasma serology was negative. Serum angiotensin converting enzyme (ACE) was $30 \mu \mathrm{L}$ (normal) and the chest X-ray was normal. Serum HIV and human leukocyte antigen (HLA) B51 were negative.

The aqueous tap result for herpes simplex polymerase chain reaction (PCR) was negative. T-lymphocyte profiling revealed a CD8 percentage of $20.6 \%$ in peripheral blood and $45.1 \%$ in the aqueous humor. The CD4/CD8 ratio was 2.13 in the peripheral blood and 1.19 in the aqueous humor.

Based on the Behçet diagnostic criteria of the international study group, a diagnosis of Behçet's uveitis was made.
The patient was started on oral prednisone $1 \mathrm{mg} / \mathrm{kg}$ per day, oral cyclosporine $100 \mathrm{mg}$ twice a day and oral azathioprine $75 \mathrm{mg}$ at night. At 6 months follow-up his right eye had counting fingers vision (navigational) while the right eye remained NLP. Uveitis was not active in either eye, and renal and liver functions and blood pressure were all normal.

\section{Discussion}

The diagnosis of $\mathrm{BD}$ depends on the recognition of multiple organ involvement, which often appears at different times in the course of the disease. There was initial hesitation in making the diagnosis of $\mathrm{BD}$ in our patient, even though he had typical mouth and penile ulcers together with skin lesions. This is largely because it is very uncommon in SSA countries. In SA, for example, only one case series has been reported on $\mathrm{BD}$ in black SA patients and none of the patients reported in this series had uveitis in their clinical course. ${ }^{[4]}$ The rarity of BD in SA black patients made it an unlikely diagnosis in our case, with conditions such as syphilis and herpes simplex infection more likely possibilities. Syphilis serology, aqueous PCR testing for herpes simplex and HIV testing were negative. The low aqueous CD4/CD8 ratio relative to peripheral blood in our patient increased our index of suspicion and helped us make the diagnosis of $\mathrm{BD}$.

A low $\mathrm{CD} 4 / \mathrm{CD} 8$ ratio has been reported in patients with $\mathrm{BD}$ uveitis before. ${ }^{[5]}$ This low aqueous CD4/CD8 ratio relative to peripheral blood is due to elevated intraocular CD8 cells. The pathogenesis of the elevated intraocular CD8 cells in Behçet's uveitis is unclear.

Intraocular CD4/CD8 ratio has been reported to be useful in the diagnosis of other uveitis entities such as sarcoidosis, ${ }^{[6]}$ in which the uveitis is mediated by CD4 cells, resulting in significantly elevated intraocular CD4 cells compared with peripheral blood. This gives rise to an aqueous $\mathrm{CD} 4 / \mathrm{CD} 8$ that is much higher than in peripheral blood.

The incidence of HLA B51 is reportedly high in patients with $\mathrm{BD}$ and yet in SSA patients, this is often negative on testing. ${ }^{[7]}$ This, together with the rarity of BD in SSA, often results in a decreased index of suspicion of $\mathrm{BD}$, resulting in its delayed diagnosis. This may, in all likelihood, result in irreversible loss of vision by the time the diagnosis is made.

Measurement of the intraocular CD4/CD8 ratio may aid in making an early diagnosis of Behçet's uveitis, particularly in countries where $\mathrm{BD}$ is uncommon. 
Acknowledgements. Dr Denise Lawrie from the Flow Cytometry Unit is acknowledged for assisting with flow cytometry.

1. Nussenblatt RB. Uveitis in Behcet's disease. Int Rev Immunol 1997;14(1):67-79. http://dx.doi org/10.3109/08830189709116845

2. International Team for the Revision of the International Criteria for Behçet's Disease. Revision of the International Criteria for Behçet's disease (ICBD). Clin Exp Rheumatol 2006;24(Suppl 42):S14-S15. International Criteria for Behçet's disease (ICBD). Clin Exp Rheumatol 2006;24(Suppl 42):S14-S15.
3. Yurdakul S, Yazici Y. Epidemiology of Behçet's syndrome and regional differences in disease expression. In: Yazici Y, Yazici H, eds. Behçet’s Syndrome. New York: Springer, 2010:35-53.

4. Jacyk WK. Behçet's disease in South African blacks: Report of five cases. J Am Acad Dermatol 1994;30(5):869-873.
5. Yu HG, Lee DS, Seo JM, et al. The number of CD8+ T cells and NKT cells increases in the aqueous humor of patients with Behçet's uveitis. Clin Exp Immunol 2004;137(2):437-443. http://dx.doi. 6. $101111 / j 1365-2249200402536 \times$

6. Kojima K, Maruyama K, Inaba T, et al. The $\mathrm{CD} 4 / \mathrm{CD} 8$ ratio in vitreous fluid is of high Kojima K, Maruyama K, Inaba T, et al. The CD4/CD8 ratio in vitreous fluid is of high
diagnostic value in sarcoidosis. Ophthalmol 2012;119(11):2386-2392. http://dx.doi.org/10.1016/j. ophtha.2012.05.033

7. Liozon E, Roussin C, Puechal X, et al. Behçet's disease in East African patients may not be unusual and is an HLA-B51 negative condition: A case series from Mayotte (Comoros). Joint Bone Spine 2011;78(2):166-170. http://dx.doi.org/10.1016/j.jbspin.2010.05.007

Accepted 29 September 2016. 